\title{
New Synthesis: Plant Volatiles as Functional Cues in Intercropping Systems
}

\author{
K. Poveda $\cdot$ A. Kessler \\ Published online: 26 October 2012 \\ (C) Springer Science+Business Media New York 2012
}

The practice of intercropping has begun to show its enormous potential to translate discoveries in chemical ecology to improve crop pest control and reduce dependence on pesticides (Hassanali et al., 2008). However, the actual mechanism by which intercropping works remains elusive, and the proposed plant-chemistry based hypothesis was recently challenged (Finch and Collier, 2012). A common view proposes that intercrops come in two functional groups, trap plants and repellent plants, which have chemical characteristics making them attractive or repellent to a certain insect pest, thereby reducing pest pressure on the main crop. To identify the exact underlying mechanisms is crucial for the broad application of intercrop pest control strategies.

The search for generalizable mechanisms, as reflected in the three studies profiled in this essay, becomes apparent when an intercrop system is as successful in controlling insect herbivores as is the push-pull approach used to control cereal pests in Africa. This approach is based on the use of behavior-modifying stimuli that reduce damage by stem boring Lepidoptera (Noctuidae, Busseola fusca or Crambidae, Chilo partellus) in maize. Maize is intercropped with the legume Desmodium uncinatum or the grass Melinis minutiflora, both of which dramatically reduce plant damage by repelling (pushing) the adult stem-borer moths out of the field, while Napier grass (Pennisetum purpureum) is planted around the field edges to attract (pull) gravid females away from maize. The combined use of these two types of stimuli reduces stem borer damage by two thirds and at least doubles maize yields in comparison with control plots (Hassanali et al., 2008).

The success of this approach has been attributed to release of attractive and repellent semiochemicals by the "push" and "pull" companion plants, respectively. Identification of specific volatile organic compounds (VOC) through GC-EAG and corroboration of their behavioral significance suggest attractive as well as repellent functions of intercrop VOCs (Hassanali et al., 2008). One major prediction of this hypothesis is that plant chemicals (e.g. VOCs) act as long-range cues that result in a non-random movement/distribution of pest insects, with reduced densities on the main crop.

Finch and Collier (2012) in a recent critique of the mechanism underlying the push-pull system, contrast the functional VOC hypothesis with appropriate/inappropriate landing theory, which assumes random herbivore movement and oviposition/consumption responses based on host plant stimuli that are experienced by insects after landing. In this view, all short-and long range physical and chemical plant characteristics are potentially important for host choice by herbivores, rendering

K. Poveda $(\bowtie)$

Entomology, Cornell University,

Ithaca, NY 15853, USA

e-mail: kap235@cornell.edu

A. Kessler

EEB, Cornell University,

Ithaca, NY 15853, USA

e-mail: ak357@cornell.edu the two hypothetical frameworks non-exclusive. Nevertheless, the controversy reveals major research questions for the future, of which two are particularly apparent:

First, Finch and Collier (2012) argue that given the vast diversity of plant VOCs, it would be maladaptive for herbivores to perceive an undefined number of non-host chemicals as repellant compounds. Although this certainly is a viable argument, it also is important to note that it may be sufficient for host-searching insects to perceive and learn volatiles associated with their hosts' quality. Hassanali et al. (2008) show that repellent plants in the African push-pull system emit at least five electrophysiologically active VOCs, of which two are produced by maize when damaged by stem-borers. The data suggest that stem borer adults perceive and avoid VOCs that are part of the host plant's VOC bouquet but function as inducible repellants because they are associated with reduced food quality. While this supports the repellant VOC hypothesis, it remains unknown if these compounds prevent pests from landing (repellency, as used by Finch and Collier) or only from ovipositing, as measured in the push-pull study (Hasannali et al., 2008).

Second, Finch and Collier (2012) suggest that intercrops hypothetically could function as physical barriers and thus "hide" the target crop from herbivores. However, in the push-pull system, the most commonly used repellent crop, $D$. uncinatum, does not overgrow maize, and by the time maize plants are suitable hosts for stem-borers, they have emerged prominently above the Desmodium intercrop canopy. Moreover, not all intercrops cause a reduction in pest density even if structurally similar. Support for this notion comes from a study in which individual maize plots were intercropped with one of four potential Desmodium species, or cowpeas (Khan et al., 2006). Maize intercropped with cowpeas had as much stemborer damage as maize without any intercrop. In contrast, maize plots with any of the four species of Desmodium suffered two thirds less damage than control plots. These data argue for a chemical over a physical mechanism of pest repulsion.

The long term push-pull project unequivocally demonstrates the power of intercropping as a pesticide-free alternative form of integrated pest management. The three papers highlighted here emphasize the need for common definitions as well as thorough investigation of the repellency function in intercrop systems to make the push-pull approach a more broadly generalizable application of chemical ecology in agriculture.

\section{References}

FINCH, S. and COLLIER, R. 2012. The influence of host and non-host companion plants on the behaviour of pest insects in field crops. Entomol. Exp. Appl. 142:87-96.

Hassanali, A., Herren, H., Khan, Z., Pickett, J., and WoOdCock, C. 2008. Integrated pest management: The push-pull approach for controlling insect pests and weeds of cereals, and its potential for other agricultural systems including animal husbandry. Phil. Trans. R. Soc. B 363:611-621.

Khan, Z., Pickett, J., Wadhamsb, L., Hassanali, A., and Midega, C. 2006. Combined control of Striga hermonthica and stemborers by maizeDesmodium spp. intercrops. Crop Protection 25:989-995. 